\title{
The extensive nature of group quality
}

\author{
R. KenNA ${ }^{1}$ and B. Berche ${ }^{2}$ \\ 1 Applied Mathematics Research Centre, Coventry University, Coventry, CV1 5FB, England, EU \\ 2 Institut Jean Lamour ${ }^{(\mathrm{a})}$, CNRS - Nancy Université - UPVM, B.P. 70239, F - 54506 Vandouvre lès Nancy Cedex, \\ France, EU
}

PACS $89.75 .-\mathrm{k}$ - Complex systems
PACS $01.75 .+\mathrm{m}$ - Science and society
PACS $05.65 .+\mathrm{b}$ - Self-organized systems

\begin{abstract}
We consider groups of interacting nodes engaged in an activity as many-body, complex systems and analyse their cooperative behaviour from a mean-field point of view. We show that inter-nodal interactions rather than accumulated individual node strengths dominate the quality of group activity, and give rise to phenomena akin to phase transitions, where the extensive relationship between group quality and quantity reduces. The theory is tested using empirical data on quantity and quality of scientific research groups, for which critical masses are determined.
\end{abstract}

Introduction. - In recent times statistical physics has found applications beyond its traditional confines and its methods have been deployed to garner new insights in many academic disciplines [1]. These include sociology, economics, complex networks [2] as well as in more exotic areas [3]. Each of these disciplines involve cooperative phenomena emerging from the interactions between individual units. Microscopic physical models - mostly of a rather simple nature - help explain how the properties of such complex systems arise from the properties of their individual parts.

Here we consider groups of interacting nodes engaged in a common activity, such as research groups of interacting scientists, as complex systems. Also in recent times, the assessment of the relative strengths of such research groups has grown in importance as universities, funding councils and governments seek to decide on where to focus investment. The debate amongst policy makers is whether to concentrate funding in relatively few well resourced institutions or to promote competition amongst a wider set, where pockets of research excellence are found [4]. A central question in this debate is whether there exists a critical mass in research, and if so, what is it?

We present a simple model which captures the complex nature of group quality as deriving both from the strengths of its members and from the interactions between them. We show the latter is, in fact, the dominant mechanism

(a) Laboratoire associé au CNRS UMR 7198 which drives the quality of group activity. Phenomena akin to phase transitions are manifest in our model and two related significant group sizes emerge: a critical mass below which a group is vulnerable to extinction and a higher value at which the correlation between group quality and quantity reduces. We test our theory using empirical data on the quality of research groups in natural sciences, for which we determine the critical masses.

Group quality and quantity. - The empirical work presented here is based upon measures of research quality as determined by the UK's Research Assessment Exercise (RAE) and the French equivalent, which is performed by Agence d'Évaluation de la Recherche et de l'Enseignement Supérieur (AERES). For the RAE, research groups were scrutinized to determine the proportion of work which fell into five quality levels from $4^{*}$ (world leading) to $1^{*}$ (nationally recognized) and unclassified. Based on the resulting quality profiles, a formula is used to determine how research funding is distributed. Assuming this to be a reasonably reliable and robust process, we consider this as a basis for a measurement of the quality of research groups. If $p_{n *}$ represents the percentage of a team's research which was evaluated as $n^{*}$, this gives that team's overall quality as $s=p_{4^{*}}+3 p_{3^{*}} / 7+p_{2^{*}} / 7$. A naive expectation is that, while larger quality values may be associated with older, more prestigious institutions, these variables are otherwise randomly distributed. Indeed, this is the picture implied by Fig. 1(a), where quality measurements are plotted al- 

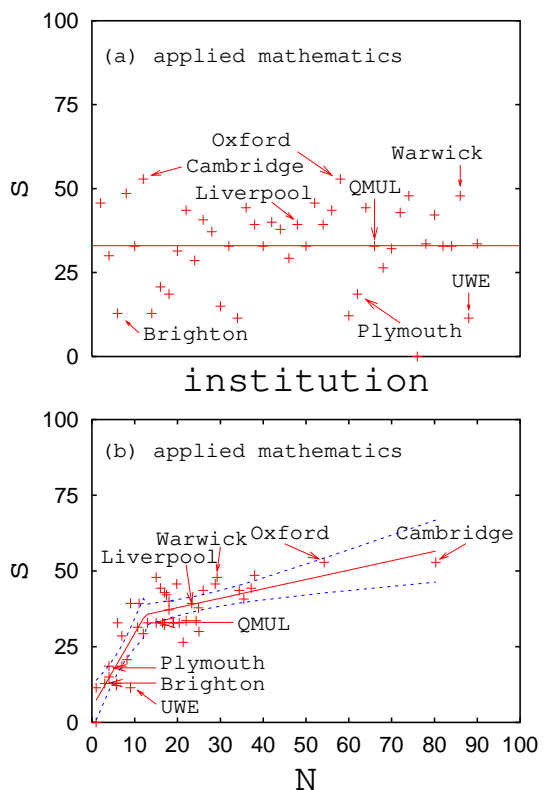

Fig. 1: Research quality $s$ for each of 45 UK applied mathematics research groups (a) arranged alphabetically and (b) as a function of group size $N$. (The sample institutions include Queen Mary, University of London (QMUL) and the University of the West of England Bristol (UWE).) In (b) the solid lines are piecewise linear regression best-fits to the data and the dashed curves represent $95 \%$ condidence intervals for these fits.

phabetically for the 45 UK applied mathematics (which includes theoretical physics) groups assessed at RAE. Such plots form the basis of rankings through which the various institutions are compared. Associated with these rankings is the notion that group quality is a measure of the average calibre of individuals forming the group, i.e., if $a_{g_{i}}$ represents the strenth of the $i^{\text {th }}$ individual in group $g$, then the strength of that group is naively given by $S_{g}=\sum_{i=1}^{N} a_{g_{i}}=N \bar{a}_{g}$, where $\bar{a}_{g}$ is the average strength of the $N$ individuals in the group. Defining the quality $s_{g}$ of the group as its average strength per head, so that $s_{g}=S_{g} / N$, one is then led to the naive conclusion that mean calibre $\bar{a}_{g}$ is given by the group quality $s_{g}$. This is the standard conclusion from a naive approach associated with Fig. 1(a). We will show that such a conclusion is dangerous and wrong.

A hint at the correct interpretation is given in Fig. 1(b), where quality measures are plotted against quantity in the form of group sizes. Clearly there is a linear relationship between quality $s$ and quantity $N$, at least in the left part of the curve. From a statistical physics point of view, there is an obvious interpretation in terms of the links between individuals. This interpretation will lead to strong and unobvious conclusions regarding the relationship between quality and quantity as well as to the quantification of the hitherto intuitive notion of critical mass in research [4].

We first consider the situation where the number of nodes $N$ in group $g$ is not too large, and where one may represent the group as a graph which is complete in the sense that it has $N(N-1) / 2$ active edges. With $b_{g_{i, j}}$ representing the strength of interaction between the $i^{\text {th }}$ and $j^{\text {th }}$ individuals, the group strength is $S_{g}=\sum_{i=1}^{N} a_{g_{i}}+$ $\sum_{\langle i, j\rangle=1}^{N(N-1) / 2} b_{g_{i, j}}=N \bar{a}_{g}+N(N-1) \bar{b}_{g} / 2$, where $\langle i, j\rangle$ represents the link between nodes $i$ and $j$ and $\bar{b}_{g}$ is the average strength of interactions between them. Inspired by molecular field theory [5], we now write the average total strength of such complete single-cluster groups as

$$
S \propto \bar{a} N+\frac{\bar{b}}{2} N(N-1)
$$

where $\bar{a}$ and $\bar{b}$ are the mean values of node and interaction strength averaged over all groups.

In fact, since two-way communication can only be carried out effectively between a limited number of nodes, it may be further expected that, as the size $N$ of a group is increased, a transition point $N_{c}$ is eventually reached, beyond which the clustering coefficient of the graph decreases. If a given node can interact meaningfully with at most $N_{c}$ others, the group may cluster into $N /\left(\alpha N_{c}\right)$ subgroups, of mean size $\alpha N_{c}$, say, within which meaningful interactions can take place. This is reminiscent of the de Gennes blob picture for polymers [6]. Mean strength of the collective due to individual strength and intra-subgroup interaction is now $S=\bar{a} N+\bar{b} N\left(\alpha N_{c}-1\right) / 2$. Each of the $N /\left(\alpha N_{c}\right)$ subgroups may interact with strength $\beta$, say, with the $N /\left(\alpha N_{c}\right)-1$ others. This inter-subgroup interaction contributes an additional strength proportional to the number of such inter-subgroup links. The mean strength of a group with $N>N_{c}$ is then

$$
S \propto \bar{a} N+\frac{\bar{b}}{2} N\left(\alpha N_{c}-1\right)+\frac{\beta}{2} \frac{N}{\alpha N_{c}}\left(\frac{N}{\alpha N_{c}}-1\right) .
$$

The size $N_{c}$ may thus be considered a transition point between "small/medium" and "large" groups. It marks the number of nodes with which an individual can cooperate in a meaningful sense.

Therefore the "mean-field" relationship between the quality of a group engaged in a given activity and its quantity or size $N$ is

$$
s=\frac{S}{N}= \begin{cases}a_{1}+b_{1} N & \text { if } N \leq N_{c} \\ a_{2}+b_{2} N & \text { if } N \geq N_{c}\end{cases}
$$

where $a_{1}, \ldots, b_{2}$ are related to the mean individual, intragroup and inter-subgroup interaction strengths. Continuity at the breakpoint requires that

$$
N_{c}=\frac{a_{2}-a_{1}}{b_{1}-b_{2}}
$$

As its size grows beyond $N_{c}$, two-way collaboration between all nodes is no longer the dominant driver of quality of the group. Interactions between subgroups may instead 

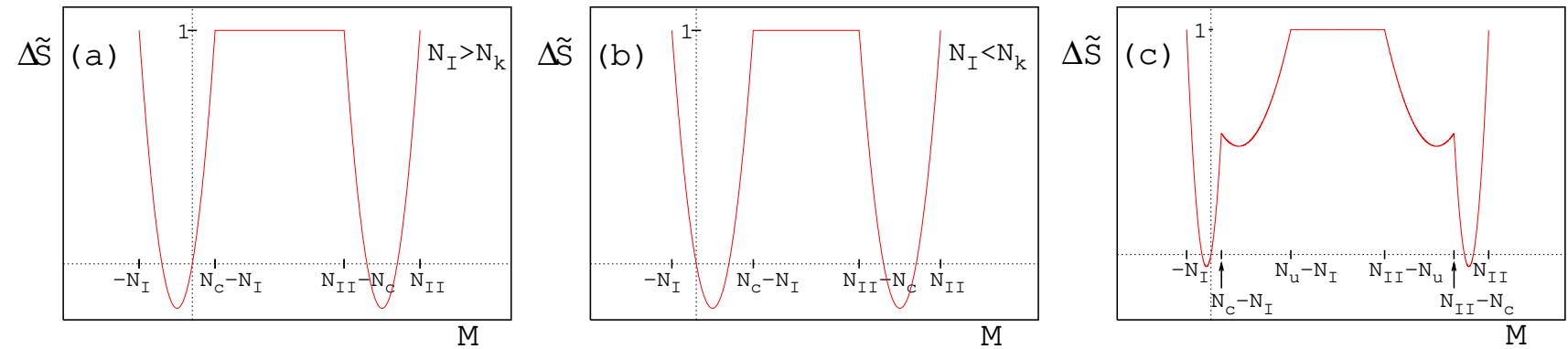

Fig. 2: Phase diagrams representing the mean total increase in strength in an activity obtained by transferring $M$ nodes from group II to group I. (a) If group I is initially supercritical, the positive gradient at $M=0$ indicates that incremental transfer to it from the large group is globally beneficial for the activity. (b) If group I is initially sub-critical the transfer of mass to it from the large group is only beneficial if a sufficient number of nodes (namely $N_{c}-N_{\mathrm{I}}$ ) is involved. (c) Phase diagram when two breakpoints are present, one at $N=N_{c}$ and the other at $N=N_{u}$.

be expected to lead to a milder dependency of $s$ on $N$. Indeed, from (2), one may expect the slope $b_{2}$ to decrease with the size of the breakpoint $N_{c}$. We shall provide empirical evidence in support of this and see that $b_{2}$ is close to zero for large $N_{c}$. Thus the slope to the right of the breakpoint $N_{c}$ plays a role similar to that of the order parameter in the theory of critical phenomena (we are not interested in the thermodynamic limit here).

Critical mass. - Having quantified the notion of large teams as those whose size exceeds the transition point $N_{c}$, we next attempt to pin down the meaning of the term critical mass. This is loosely described as the value $N_{k}$ of $N$ beneath which groups are not viable in the longer term. We refer to teams of size $N<N_{k}$ as small and groups with $N_{k} \leq N<N_{c}$ as medium in size. To determine $N_{k}$, we consider groups as canonical or grand canonical ensembles.

In the latter case, one may ask, if new nodes become available, is it more beneficial for the strength of the entire society in the given activity to allocate them to a small, medium or large team? From (3), the gradient of $S=s N$ is $a_{j}+2 b_{j} N$, where $j=1$ or 2 according to whether $N<$ $N_{c}$ or $N>N_{c}$. The former has greater value, indicating it is more beneficial if these new nodes are allocated to the small/medium group, provided $a_{1}+2 b_{1} N>a_{2}+2 b_{2} N$ or $N>N_{k}$, where $N_{k}$ is given by the scaling relation

$$
N_{k}=\frac{a_{2}-a_{1}}{2\left(b_{1}-b_{2}\right)}=\frac{N_{c}}{2}
$$

having used 4. The quantity $N_{k}$ may then be considered the critical mass for the given activity.

For the canonical approach, we may ask the complementary question: if the total number of nodes associated with the given activity is fixed, what is the best strategy, on average, for transferring them between small/medium and large groups? We suppose that group I initially has

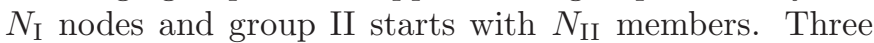
scenarios arise. In scenario A, group I is small/medium and group II is large; in scenario B, both groups are large and Scenario $\mathrm{C}$ is the inverse of Scenario $\mathrm{A}$ and needs no further consideration. We consider the transfer of $M$ nodes from group II to group I. Starting with Scenario A, the post-transfer strength of the new configuration is given by $S_{i}(M)=a_{i}\left(N_{\mathrm{I}}+M\right)+b_{i}\left(N_{\mathrm{I}}+M\right)^{2}+a_{2}\left(N_{\mathrm{II}}-M\right)+$ $b_{2}\left(N_{\mathrm{II}}-M\right)^{2}$, where $i=1$ or $i=2$ for scenarios $\mathrm{A}$ and $\mathrm{B}$ as the final configuration, respectively. Measuring the total increase in strength due to the transfer by

$$
\Delta \tilde{S}(M)=\frac{S_{i}(M)-S_{1}(0)}{b_{1} N_{\mathrm{I}}\left(N_{c}-N_{\mathrm{I}}\right)},
$$

and plotting this in Fig. 2(a) and (b), up to corrections of order $b_{2}$, two outcomes emerge. For scenario A the collective increase $\Delta \tilde{S}$ is maximized at $M=-N_{\mathrm{I}}$, which represents the assimilation of group I into group II. For scenario $\mathrm{B}$, one finds that this maximum holds for $N_{c}-$ $N_{\mathrm{I}} \leq M \leq N_{\mathrm{II}}-N_{c}$, which represents any configuration in which each group has at least $N_{c}$ members.

Incremental transfer of staff from group II to group I is governed by scenario A, the outcome of which is determined by the gradient of $\Delta \tilde{S}$, at the initial point $M=0$,

$$
\left.\frac{\partial \Delta \tilde{S}}{\partial M}\right|_{M=0}=\frac{2}{N_{\mathrm{I}}} \frac{N_{\mathrm{I}}-N_{k}}{N_{c}-N_{\mathrm{I}}} .
$$

Such a move is globally beneficial if this slope is positive. This occurs when $N_{\mathrm{I}}>N_{k}$, so that group I is initially supercritical in size. I.e., it is sensible to promote mediumsize groups at the expense of large ones. If $N_{\mathrm{I}}<N_{k}$ (group I is initially subcritical), the shortest route to maximal performance is to conglomerate all nodes into the large group. Instead, and to avoid extinction, group I should strive to achieve critical mass, in which case the previous argument prevails.

Application to research groups. - To test our theory, we require a collection of small, medium and large groups engaged in an activity in which the quality of each 

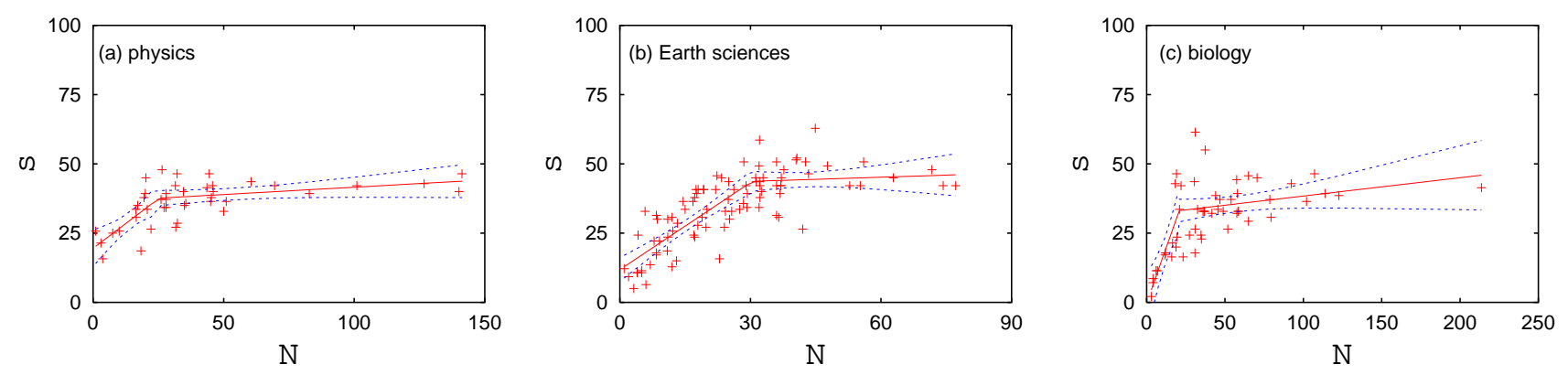

Fig. 3: Success rate or research quality $s$ as a function of group size $N$ for (a) physics, (b) Earth science and (c) biology. As in Fig.1(b), the solid lines are piecewise linear regression best-fits to the data and the dashed curves represent $95 \%$ condidence intervals for these fits.

group is measured quantitatively. As mentioned, the UK's academic communities provide suitable testing arenas as the quality of research groups in many disciplines has been measured through RAE.

A piecewise linear regression analysis is applied to data sets corresponding to measured quality of research groups, fitting to the form (3). In Fig. 3 the data for physics (which includes experimental physics), Earth sciences and biology are presented. For these, as in Fig. 1(b) for applied mathematics, the positive correlation between quality and team size reduces beyond a discipline-dependent transition point. The statistical $P$-values for the null hypothesis that there is no underlying correlation were less than $0.1 \%$ in each case while the $P$-values for coinciding slopes on either side of the transition point are smaller than $0.3 \%$. These small values indicate that we can reject these hypotheses for these disciplines, in favour of the alternative that the model is sound and that the transition points exist. The solid curves in the figures are the piecewise linear-regression fits to the data and the dashed curves represent the resulting $95 \%$ confidence belts. For physics, we estimate the critical mass as $N_{k}=12.7 \pm 2.4$, about twice the estimate $N_{k}=6.2 \pm 0.9$ for applied mathematics and closer to the estimate $N_{k}=15.2 \pm 1.4$ for Earth sciences and to $N_{k}=10.4 \pm 1.6$ for biology.

The measured values for the slopes to the right of the breakpoint are $b_{2}=0.05 \pm 0.03,0.05 \pm 0.11$ and $0.07 \pm 0.04$ for physics, Earth sciences and biology, respectively. As expected for large- $N_{c}$ communities, these slopes are small compared to that of applied mathematics, which has $b_{2}=$ $0.3 \pm 0.1$ and a relatively small value of $N_{c}$.

In pure mathematics (see Fig. 4(a)) no transition point was detected and the data is best fitted by a single line, the intercept $(28.1 \pm 2.8)$ and slope $(0.5 \pm 0.2)$ of which are comparable to the corresponding values for large applied mathematics groups $\left(a_{2}=31.7 \pm 12.8\right.$ and $0.31 \pm 0.09$, respectively). These indicate that these data may also be interpreted as belonging to large groups. Then $N_{c}$ for pure mathematics may be interpreted as being less than or equal to the size of the smallest group whose quality was measured, which was 4 , so that $N_{k} \leq 2$. This suggests that local cooperation is less significant in pure mathematics, where the work pattern is more individualized. This is consistent with experience: papers in pure mathematics tend to be authored by one or two individials, rather than by larger collaborations. The results for chemistry are presented in Fig. 4(b), where there is also an absence of small groups leading to a relatively large error in the critical mass estimate $N_{k}=18 \pm 7$.

The above work is based upon the measures of research quality as determined in the UK. To check its broader generality, we compare the results of the RAE with those of the French equivalent, which is performed by the Agence d'Évaluation de la Recherche et de l'Enseignement Supérieur (AERES). In the 2008 evaluation, a method was used which is considered more precise than that used previously and this facilitates comparison with the British approach. However, since only 10 traditional universities were evaluated, the amount of data available for the French system is lower than for the UK equivalent. Furthermore, only a global mark is attributed to cumulated research groupings so a fine-grain analysis at the level of the research groups is not possible and no transition point is measureable. Nonetheless, we translate the AERES grades $\mathrm{A}+, \mathrm{A}, \mathrm{B}, \mathrm{C}$ into $4^{*}, 3^{*}, 2^{*}$, and $1^{*}$ and analyse the French system for hard sciences and life sciences to compare to the British equivalent. In Fig. 5, the standardized success rates $\sigma=(s-\bar{s}) / \sigma_{s}$ are plotted against the standardized group sizes $\nu=(N-\bar{N}) / \sigma_{N}$ for both systems, where $\bar{N}$ and $\bar{s}$ are the mean $N$ and $s$ values, respectively, and $\sigma_{N}$ and $\sigma_{s}$ are their standard deviations. A convincing degree of overlap is evident.

Palla et al. analysed international networks of coauthorship in condensed matter physics and found a difference in the endurance rates of teams with above about 20 members and teams with fewer members [7]. Endurance over time is not the same as quality, but it may serve as a rough guide in the sense that unsuccessful research collaborations are unlikely to endure and vice versa. It is therefore satisfactory to note that our estimate $N_{c}=25.4 \pm 4.8$ for physics is consistent with the observation of Ref. [7]. 

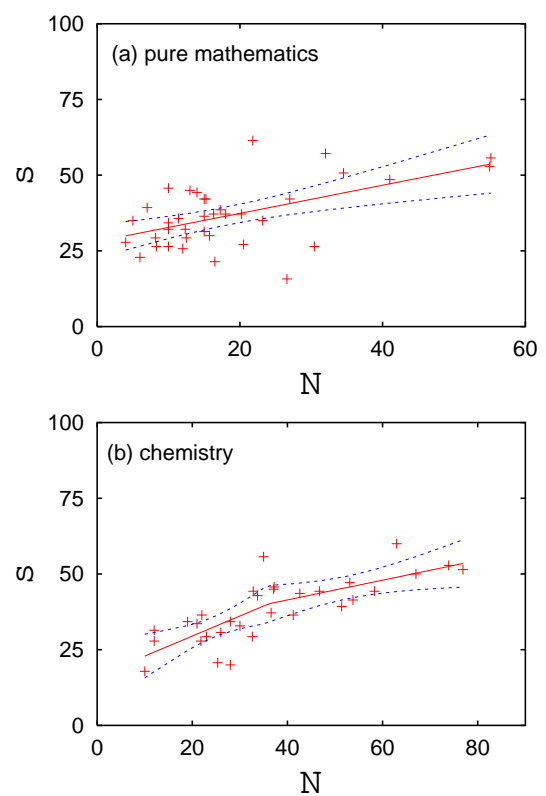

Fig. 4: Assessed group quality as a function of size for (a) pure mathematics and (b) chemistry. The former is best described by a single straight line.

Discussion. - In analysing the relationship between quality in an activity and quantity of parcipants, we have considered communities as consisting of individuals collected as subgroups within groups. It is a simple matter to extend these considerations to ever larger collectives, formed from further hierarchies of communities with self-similar interactions between them. In the case where $b_{2} \neq 0$, the quality plateau may occur at a larger value $N=N_{u}$, which marks the point where inter-subgroup interactions saturate. In this case, (3) is replaced by

$$
s= \begin{cases}a_{1}+b_{1} N & \text { if } N \leq N_{c} \\ a_{2}+b_{2} N & \text { if } N_{c} \leq N \leq N_{u} \\ a_{3} & \text { if } N \geq N_{u} .\end{cases}
$$

This double-breakpoint scenario leads to a richer phase diagram, obtainable by consideration of transfer of $M$ nodes from group II, of size $N_{\text {II }}>N_{u}$. This is plotted in Fig. 1(c) for a super-critical group I. In Ref. [8], where a study of the hierarchical structure of nested communities in a university network indicated self-organization into a self-similar structure, it was suggested that some universal mechanism could be behind the evolution of social networks. The mechanism we propose is of this type.

We have shown that there is a somewhat surprising relationship between quality and quantity: in the language of statistical physics, quality is an extensive concept. Our study also shows that it is unwise to judge a research group solely on the basis of its quality profile - precisely because of this strong size dependency: small- and medium-size groups should not be expected to yield the same quality profiles as large ones, and to compare small/medium
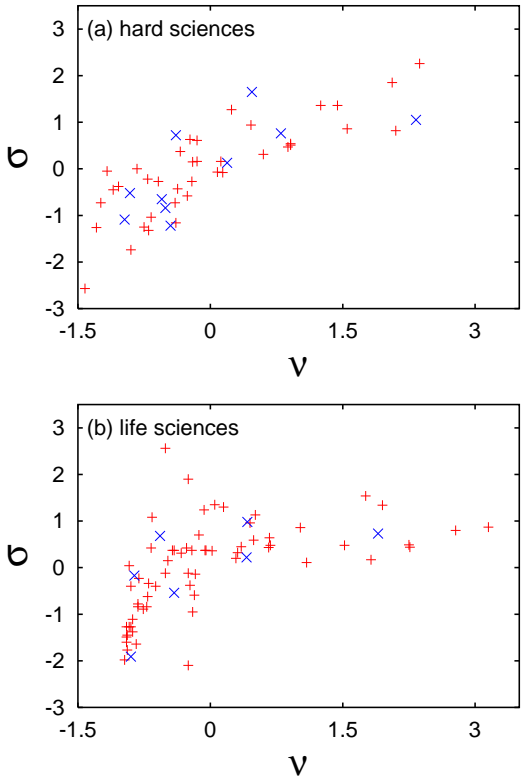

Fig. 5: Standardized success rates $\sigma=(s-\bar{s}) / \sigma_{s}$ plotted against standardized sizes $\nu=(N-\bar{N}) / \sigma_{N}$ for France's AERES and the UK's RAE for (A) the hard sciences and (B) the life sciences. The French data correspond to the symbols $\times$ (blue online) and the integrated British data to + (red online).

groups to the average success rate over all research groups in a discipline can be misleading. Indeed, Fig. 6 contains a plot of renormalized research quality measures $s-\langle s\rangle$, where $\langle s\rangle$ is the $N$-dependent expected quality value from Eq.(3). The standard deviation and range corresponding to this plot are 6.4 and 28.8, respectively. These values are about half the corresponding values of 12.6 and 52.9 , respectively, for Fig. 1(a), where the data are compared to their global average. Similar results are obtained for other disciplines.

Our model is based on the notion that research groups are complex systems and scientific quality is dominated by interactions between group members rather than by the accumulated node strengths. While the data analysis supports this hypothesis, one could, of course, consider other possible mechanisms which drive the quality of group activity, such as preferential recruitment of quality to quality (allowing for the average quality of individuals at some institutions to be higher than at others) or one in which increased quality drives increased quantity (the opposite causal mechanism to that of our model). Or there could be a bias in both the RAE and AERES where assessment of research may favour larger groups. However, one would expect such mechanisms to lead to a sustained increase of quality with quantity and it is therefore hard to see how these alone could account for the existence and properties of the breakpoint, which we have clearly established through our statistical analyses. Moreover, similar analyses of other fields confirm the existence of a breakpoint 


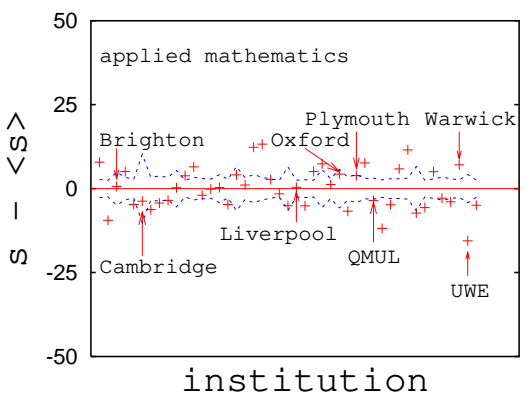

Fig. 6: The renormalized research quality $s-\langle s\rangle$ for applied mathematics. The standard deviation and range corresponding to this plot are about half those corresponding to Fig.1(a).

in areas far from the natural sciences reported here, e.g., European languages, archaelogy, philosophy and theology, which are unlikely to have the same assessment traditions or bias as the hard or life sciences. Of course, improvement of the model could introduce noise and bias, but very interestingly, these effects are an order of magnitude below the basic collaborative effect.

An analysis of the type presented herein may therefore assist in the determination of which groups are, to use a boxing analogy, punching above or below their weight within a research arena and should be taken into account by decision makers when comparing research groups and when formulating strategy. Furthermore, having established a correlation between quality and quantity, and ascribing this correlation as primarily due to two-way communication links, it is clear that facilitation of such communication should form an important management policy in academia. Indeed we have demonstrated that to optimize overall research quality in a given discipline, mediumsize groups should be promoted while small ones must endeavour to attain critical mass.

To summarize, inspired by mean field theory and the de Gennes polymer picture, we have developed a very simple model which relates the quality of an activity to the quantity of participants engaged in it. In spite of its simplicity, this self-similar model captures the essential features of the empirical data. It involves the treatment of collectives as complex systems and cooperative behaviour arising from inter-nodal interactions dominate group quality. Furthermore, these hierarchical interactions drive "phase transitions" between what may be considered small, medium and large groups and lead to a rather rich phase structure. In particular, quality saturates beyond a certain group size which is related by a simple scaling relation to a critical mass, beneath which a group is vulnerable. Considering academia as an example of an activity where quality measurements are readily available and where quantities of participants are known, we present saturation points and critical masses for research areas in a number of scientific disciplines. Critical masses for other subjects area will be presented elsewhere.

\section{$* * *$}

We thank Neville Hunt for inspiring discussions and for help with the statistical analyses. We also thank Arnaldo Donoso, Christian von Ferber, Housh Mashhoudy and Andrew Snowdon for comments and suggestions. We are grateful to Claude Lecomte, Scientific Delegate at the Agence d'Évaluation de la Recherche et de l'Enseignement Supérieur, for discussions on the work of that agency.

\section{REFERENCES}

[1] Galam S., Physica A 336 (2004) 49.

[2] Dorogovtsev S.N., Mendes J.F.F., Evolution of networks (Oxford Univ. Press, Oxford) 2003.

[3] Bittner E., Nußbaumer A., Janke W. and Weigel M., EPL 78 (2007) 58002.

[4] Harrison M., in The Question of REDD Specialisation: Perspectives and Policy Implications, edited by D. Pontikakis, D. Kriakou and R. van Baval, JRC Technical and Scientific Reports (European Commission, Luxembourg) 2009, p.53.

[5] Weiss P., J. Phys. 6 (1907) 661.

[6] de Gennes P.-G., Scaling concepts in polymer physics (Cornell Univ., Ithaca, NY) 1979.

[7] Palla G., Barabási A.-L., Vicsek T., Nature 446 (2007) 664 .

[8] Guimerà R., Danon L., Díaz-Guilere A., Giralt F. and Arenas A., Phys. Rev. E 68 (2003) 065103(R). 